\title{
Collaborative Hands-on Training on Haptic Simulators
}

\author{
Angel R. Licona R. ${ }^{1}$, Fei Liu ${ }^{1}$, Arnaud Lelevé ${ }^{1, *}$, Damien Ebérard ${ }^{1}$, and Minh Tu Pham $^{1}$ \\ ${ }^{1}$ Université de Lyon, INSA Lyon, Ampère Laboratory, Villeurbanne, F69621, France
}

\begin{abstract}
Medical trainees are required to acquire sufficient skills before touching a real patient. Nowadays, haptic simulators provide an effective solution but they do not facilitate an active supervision by a trainer who should show the right gestures in terms of motions and forces to apply, in the simulated environment. Dual user training systems aim at this purpose. Even though they permit a cooperative training, they generally dot not enable efficient demonstration/evaluation modes where the user who observes the person performing a manipulation is also able to feel the interaction forces, not only the motion. In [1], we introduced the Energy Shared Control (ESC) architecture aiming at providing the latter function. It is modeled with the Port Hamiltonian framework and it embeds a Time Domain Passivity Controller, to compose a one degree-of-freedom (dof) dualuser haptic system for hands-on training. In this paper, we extend it to three dof with three identical haptic devices. Experiments bring information about its performance.
\end{abstract}

\section{Introduction}

Medical trainees are required to acquire sufficient skills before touching a real patient. To this end, they traditionally use simulators such as "black boxes", manikins, animals and cadavers, which have the drawbacks of requiring to be prepared on demand, being hardly realistic and raising ethical issues. Recently, Virtual Reality computer based training simulators have appeared as a complementary solution. They allow trainees to practice anytime on several kinds of body parts that can be parameterized in shape, weight, disease, ... Hence, trainees can repeat a procedure in a virtual environment several times without getting short of supplies. They also can get rapid quantitative feedback instead of a qualitative assessment (such as OSATS $^{1}$ ), to figure out which skills they need to improve [2].

Nevertheless, medical trainees also need haptic feedback for an efficient learning, especially with the gestures that involve kinesthetic feeling [3]. Kinesthesia is the ability to sense the movement and position of the body limbs. Thanks to it, haptic feedback enhances training efficiency of advanced tasks, compared to training simulators providing only visual feedback. Such haptic simulators are now available on the market and are used in various medical areas [4].

However, haptic training simulators are complex and expensive. They are still not affordable on demand for medical students as the cost of medical training laboratories can rise up to US $\$ 1,000,000$ [5]. Moreover, these systems are not expandable: they cannot be simultaneously used by several users nor host several slave environments. A solution to reduce this cost is to raise the number of

\footnotetext{
*e-mail: arnaud.leleve@insa-lyon.fr

${ }^{1}$ Objective Structured Assessment of Technical Skills
}

users per simulator, which would open the way to parallel hands-on training where an instructor can teach hands-on procedures to several trainees at the same time. Furthermore, symmetrically, a trainee could have his skills simultaneously assessed by several evaluators. Trainees could thus get both a quantitative feedback from the simulator and a qualitative feedback from several evaluators.

To do so, it is necessary to spread multilateral haptic training systems in the medical schools. Various architectures have been proposed for the control of dual-user haptic systems. Next section sums up the scientific advances in this domain. As, at the start of this project, none provided a complete haptic demonstration/evaluation mode allowing the following user to reproduce the right motion realized by the leading user and to feel the same efforts during an interaction between the slave tool and its environment, we proposed in [1] an Energy Shared Control (ESC) architecture, based on an energetic modeling. It was introduced and experimentally validated for only one degree of freedom (dof). In this paper, we present its extension to three dof with three identical haptic devices and an experimental validation in the case of three dof.

This paper is organized as follows. Section 2 provides a global view about the research works on dual user training systems. Section 3 recalls the main principles of the control architecture developed in this project and its extension to three dof. Section 4 depicts its validation through experimentation.

\section{State of the Art}

A multilateral haptic training simulator is a system simultaneously manipulated by two or more users through haptic devices (masters), in order to perform a supervised 


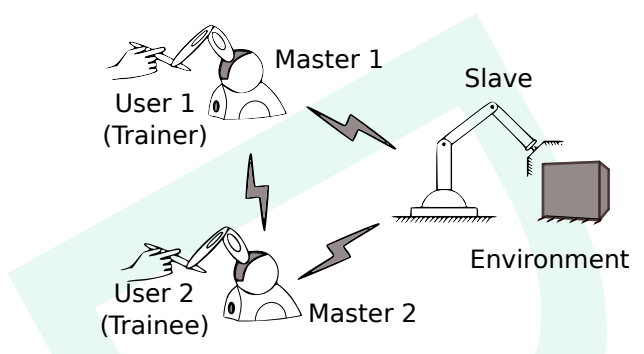

Figure 1: Dual user haptic system in a training context [1]

(guided) training of a procedure performed on one or several (real or virtual) slave device(s) in interaction with their own (and potentially shared) environment. A trainer manipulates his own haptic device and the trainees manipulate their own one. All the devices are interconnected via a controller which dispatches the flows. Fig. 1 shows a dual-user (also named trilateral) system: with one trainer, one trainee and one slave. Master 1 is the device used by the trainer and Master 2 is used by the trainee. This paper deals with such a dual user haptic training system.

As both users interact with the system at the same time, an authority sharing mechanism is mandatory. It is necessary to set $\alpha$ which tells how much dominance each user has over the slave during the manipulation [6]. The value of $\alpha$ can vary between 0 and 1, leading to three possible scenarios in a training context:

Demonstration mode $(\alpha=1)$ : the trainer fully leads the slave. The trainee follows him by observing the trainer's motions and interaction forces.

Guidance mode $(0<\alpha<1)$ for cooperative training. $\alpha$ is manually set by the trainer in real-time to adjust the dominance between both users.

Evaluation mode ( $\alpha=0$ ): the trainee has full dominance over the slave, in opposition to the demonstration mode.

In [7], Khademian et al. introduced two architectures: the Complementary Linear Combination (CLC) architecture and the Masters Correspondence with Environment Transfer (MCET). We showed in a simulation based comparative study, detailed in [8], that the ESC architecture provided similar performances as CLC and MCET, in terms of position tracking. These two architectures were chosen for their best (but not full) accordance with the hands-on training requirements recalled in the introduction, as far as we could find in the literature.

The extension of such trilateral systems, when users are remotely located, has been investigated. This case induces communication delays that greatly destabilize and drastically reduce the transparency of such architectures. In [9], Ghorbanian et al. proposed an architecture considering nonlinear teleoperator dynamics and bounded variable time-delays. More recently, Zakerimanesh et al. proposed to consider also the actuator saturations [10] (they only validated this proposal in simulation so far).

All these architectures provide very interesting results but have one drawback: during an interaction between the slave tool and its environment, when $\alpha=1$, Master 2 does not allow the following user to feel the right motion realized by the leading user and to reproduce the same efforts felt by the leader all at once. Therefore, they do not fully comply with hands-on training requirements exposed in this paper introduction.

\section{Control Architecture}

\subsection{One-DoF Interconnection architecture}

In order to provide force feedback to both users even when they have no dominance over the slave, the ESC architecture depicted in Fig. 2 has been introduced by Fei Liu et $a l$ in [1]. It is based on a one dof dual-user architecture with an energetic modeling using Port Hamiltonian theory and a Time Domain Passivity Controller (TDPC) to ensure that the system remains passive. This architecture embeds three Intrinsically Passive Controllers (IPC, introduced by Stramigioli et al in [11]) to provide compliance to the user haptic interfaces and to insure that the interfaces with users (on masters' side) and the environment (on slave side) remain independently passive. In the ESC, the slave IPC is a variant of the masters' IPC as it is controlled in torque (versus velocity for the masters). This arbitrary choice of causality provides a better transparency in practice.

Design details are provided in [1] and [8]. Figure 2 depicts this architecture. The inputs/outputs of this architecture are angular velocities $\left(\dot{\theta}_{m 1}\right.$ and $\dot{\theta}_{m 2}$ for the master interfaces and $\dot{\theta}_{s}$ for the slave) and torques ( $T_{h 1}$ and $T_{h 2}$ are applied by the users on the master haptic devices and $-T_{e}$ is the torque applied by the environment on the slave tool). $D_{m 1}, D_{m 2}$ and $D_{s}$ (in green) are lossless leveraging interconnections (Diracs) to perform authority sharing (modeled as skew-symmetric matrices in Eq. (1) and (2). They control the energy exchanged between both the masters and the slave devices according to $\alpha$.

$$
\begin{aligned}
D_{m 1}:\left[\begin{array}{c}
\dot{\theta}_{r 1} \\
T_{s 1} \\
T_{s f 1}
\end{array}\right] & =\left[\begin{array}{ccc}
0 & \alpha & 1-\alpha \\
-\alpha & 0 & 0 \\
\alpha-1 & 0 & 0
\end{array}\right]\left[\begin{array}{c}
T_{r 1} \\
\dot{\theta}_{s 1} \\
\dot{\theta}_{s f 1}
\end{array}\right] \\
D_{m 2}:\left[\begin{array}{c}
\dot{\theta}_{r 2} \\
T_{s 2} \\
T_{s f 2}
\end{array}\right] & =\left[\begin{array}{ccc}
0 & 1-\alpha & \alpha \\
\alpha-1 & 0 & 0 \\
\dot{\theta}_{s 1} \\
-\alpha & 0 & 0
\end{array}\right]\left[\begin{array}{c}
T_{r 2} \\
\dot{\theta}_{s 2} \\
\dot{\theta}_{s f 2}
\end{array}\right] \\
D_{s}:\left[\begin{array}{c}
0 \\
\dot{\theta}_{s 2} \\
T_{r s}
\end{array}\right] & =\left[\begin{array}{ccc}
0 & 0 & 1-\beta_{1} \\
T_{s 1} \\
T_{s 2} \\
\dot{\theta}_{r s}
\end{array}\right]
\end{aligned}
$$

$$
\beta_{1}=\left\{\begin{array}{ll}
\alpha, & \alpha=1,0 \\
1, & 0<\alpha<1
\end{array} \quad \beta_{2}= \begin{cases}\alpha, & \alpha=1,0 \\
0, & 0<\alpha<1\end{cases}\right.
$$

The advantage of this approach is to provide a model linking passive blocks together, which results in a global passive system. The IPC controllers feature several parameters to tune their compliance to supply the best global transparency without compromising the overall passivity. They do not introduce any steady state error (angular velocity and torque) and they permit to dispense with the estimation of the command torques from motor currents. Yet, each IPC feeds back a torque including interaction, gravity, friction effects and some other dynamic disturbances, as a global external torque. As we do not have 


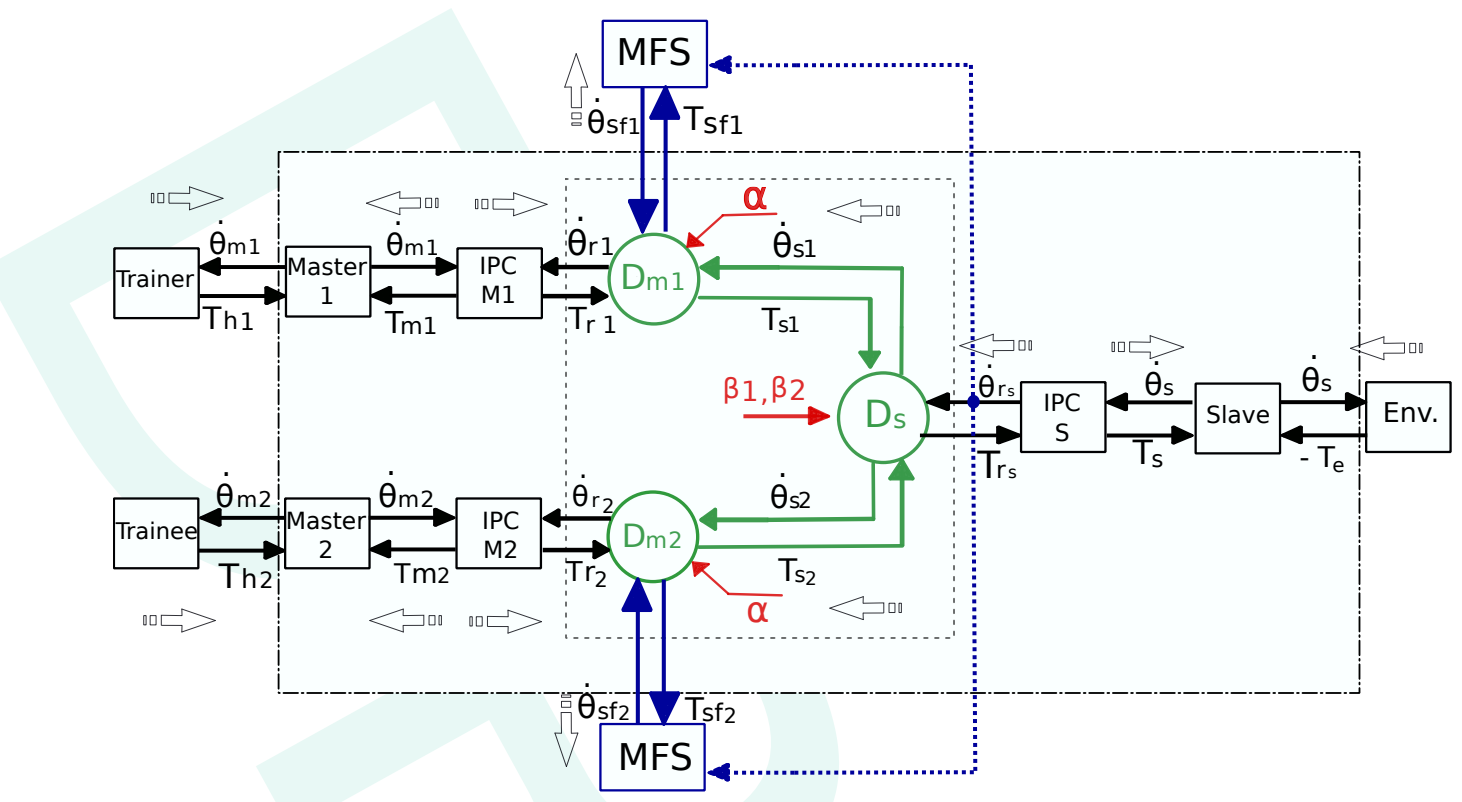

Figure 2: ESC architecture dual-User haptic system for one dof [1]

any force sensor to measure the real interaction forces, the global system control is performed with these disturbed torques. Nevertheless, as the three devices use the same equipment and have very close motions, these disturbances are nearly the same for the three of them so that, they offset one another and the leading user only feels the tool-environment interaction forces $\left(T_{r 1} \approx T_{r s} \Rightarrow\right.$ $T_{h 1} \approx T_{e}$ in practice). To filter this noise, one can install costly force sensors on each device effector or provide an estimator such as in [12], and update the IPC model to guarantee its passivity. The approach adopted in this paper limits the precision of this architecture but has the advantage of being scalable with more users or slave robots without dramatically raising its complexity.

By default, the $D_{m 1, m 2, s}$ interconnection has an important limitation in demonstration $(\alpha=1)$ and evaluation $(\alpha=0)$ modes: the following user does not get any feedback from the slave, for energy balancing reasons. A solution, proposed in [1], consists of adding 2 Modulated Flow Sources (MFS) providing the necessary complementary energy to the following device. To ensure the global passivity preservation that these additional sources of energy could compromise, a Time Domain Passivity Observer (TDPO, evaluating (3)) measures the energy $E_{p}(t)$ injected by the two MFS into the system:

$$
E_{p}(t)=\int_{0}^{t}\left[(\alpha-1) T_{r 1}(\tau)-\alpha T_{r 2}(\tau)\right]^{T} \dot{\theta}_{r s}(\tau) d \tau
$$

A conservative solution to preserve the system passivity (and therefore stability) consists in ensuring that $E_{p}(t) \leq 0$. The outputs of the MFS are accordingly modulated (4), acting as a Switched Time Domain Passivity Controller:

$$
\dot{\theta}_{s f 1}(t)=\dot{\theta}_{s f 2}(t)=\left\{\begin{array}{cc}
\dot{\theta}_{r s}(t), & \text { if } E_{p}(t) \leq 0 \\
0, & \text { otherwise }
\end{array}\right.
$$

In practice, with a standard passive environment (free or static "walls"), no passivity compromise has been observed so far.

\subsection{Extension to $\mathrm{n}$-DoF}

To create a functional simulator, the aforementioned one dof architecture has been expanded by duplicating it in parallel, independently for each joint (see Fig. 3 with three dof). Obviously, this architecture can only work when identical devices are used for masters and slave. We raised the assumption that the robustness of each IPC controller would compensate for the potential interactions between each dof. Next section details the experimental setup and results.

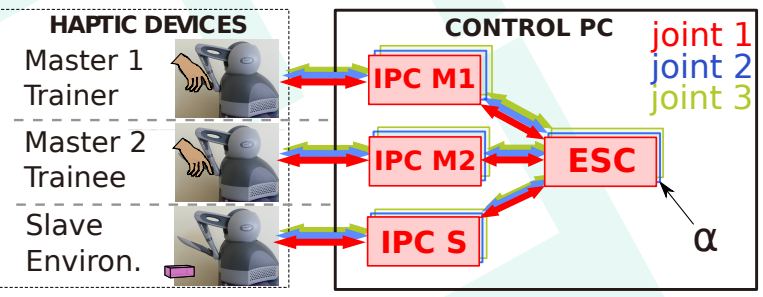

Figure 3: Multiple dof Articular ESC architecture, here with three dof

\section{Experimental Validation}

The setup is composed of three Geomagic 3D Touch ${ }^{\mathrm{TM}}$ haptic devices (see Figure 4). These devices are 6 dof systems but only three are actuated. Therefore, we immobilized the three non-actuated joints. Fig. 5 shows the Geomagic $3 \mathrm{D}$ Touch $^{\mathrm{TM}}$ system with the considered degrees 


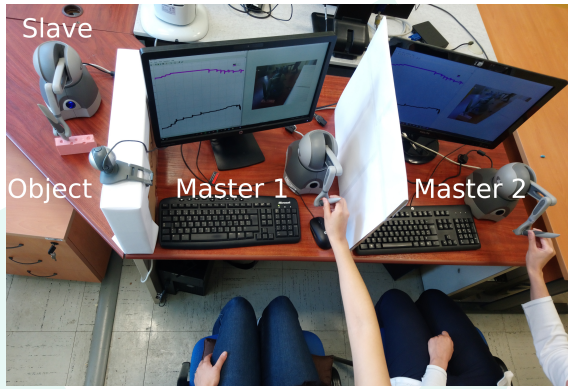

Figure 4: Setup organisation

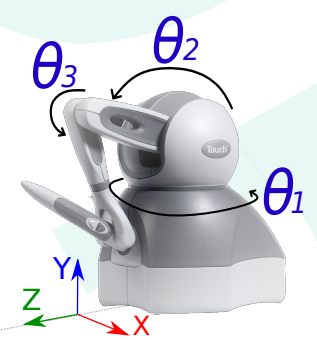

Figure 5: Geomagic 3D Touch ${ }^{\mathrm{TM}}$

between the devices and the control computer, the Open Haptics $囚$ software library was used. The control environment was implemented in Matlab Simulink $($ on a single computer. The interconnection between the Open Haptics library and Simulink was performed by the Phantorque block introduced in [13]. During all the tests, the real time clock was provided by this block. Every IPC (masters and slave, independently for each joint) was tuned to provide the best position and force tracking performance, according to [14].

\subsection{Free Motion}

In this first experiment, we set the dominance factor $\alpha$ to 1 at first and changed it to 0 at $t \approx 11.5 \mathrm{~s}$ in order to show demonstration and assessment modes, and also the effect of a sudden change of $\alpha$. Also, in order to show that the device of the following user leads him on the track of the leader, each user performed a gesture to take his device away from the prescribed trajectory (the trainee in demonstration stage a and then the trainer during the assessment stage b in Figures 6 and 7).

Figure 6 depicts the global trajectory performed by the users in free motion. This plot shows that, out of the two attempts to take the device away, the three trajectories follow close trajectories. The root mean squared (RMS) value of the tracking error (computed from the positions expressed in the cartesian space) between the Master 1 and slave devices during demonstration mode was $8.2 \mathrm{~mm}$ (and $6 \mathrm{~mm}$ between the Master 2 and the slave during demonstration mode). This level of precision is to be taken into account when choosing training applications. It can suffice for first hands-on trainings but may not be precise enough for trainings requiring more precision. To enhance the precision, we would recommend to use more powerful and precise haptic devices which could in turn permit to raise the gains of the IPC and also enhance the precision of the whole system.

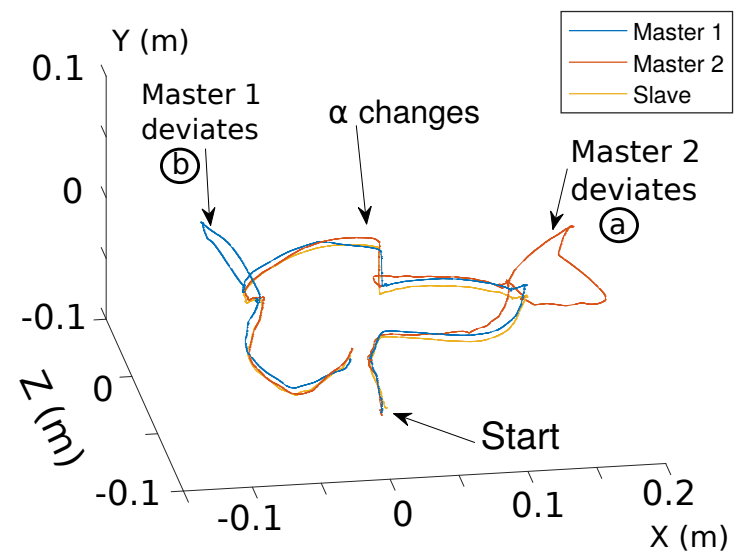

Figure 6: Projection in space of the trajectory performed in free motion
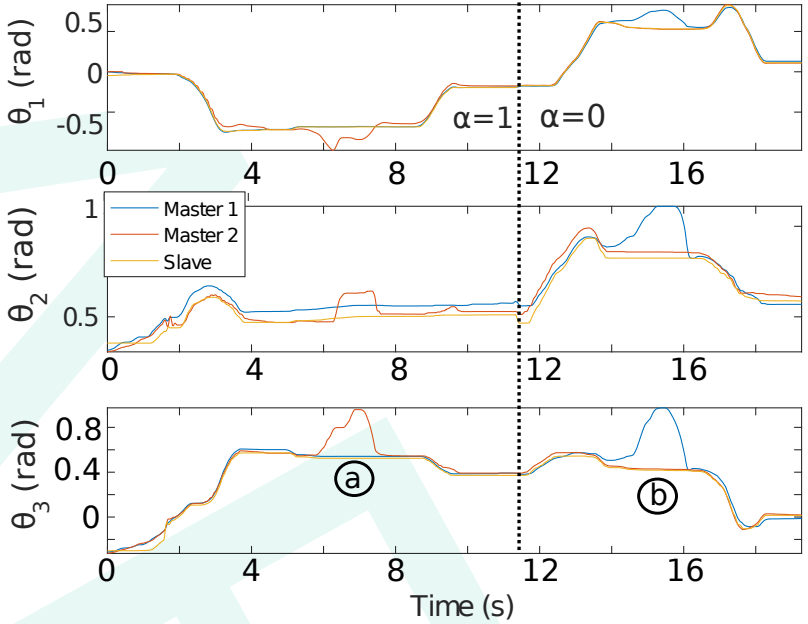

Figure 7: Angular positions for the trajectory performed in free motion

Figure 7 shows the articular positions of the three actuated joints. One can observe that the precision is the best for $\theta_{1}$ (tracking error of $17.10^{-3} \mathrm{rad}$ RMS between Master 1 and slave when $t<5 \mathrm{~s})$ and the worst for $\theta_{2}\left(55.10^{-3}\right.$ rad RMS). This can be explained by the weight of the arm which disturbs the IPC controller. A weight compensation approach should be envisaged to raise the precision of this joint control. Besides, it is logical that the follower user position does not overlap with the two others as this user is led by their device to follow the slave trajectory. According to the resistance they brings (the stiffness of their hand), the follower position is shifted from the reference trajectory.

Figure 8 shows the exchanged torques $T_{r 1,2, s}$ for the three actuated joints. The three plots show a good torque tracking for the three joints. As the motion is performed in free motion, these torques should be close to zero if 4 neglecting the aforementioned disturbances. Indeed, in 
demonstration (resp. assessment) mode, the master 1 (resp. master 2) and slave torques overlap. Moreover, one can observe the torque generated by the devices to bring back the following user to the reference trajectory at marks a and (b. Also, the sudden change of $\alpha$ results in a small sudden change of $T_{1}$ and $T_{2}$ but in practice it is hardly perceived by users. It has not any negative effect on energy levels (see Figure 9) which remain negative.

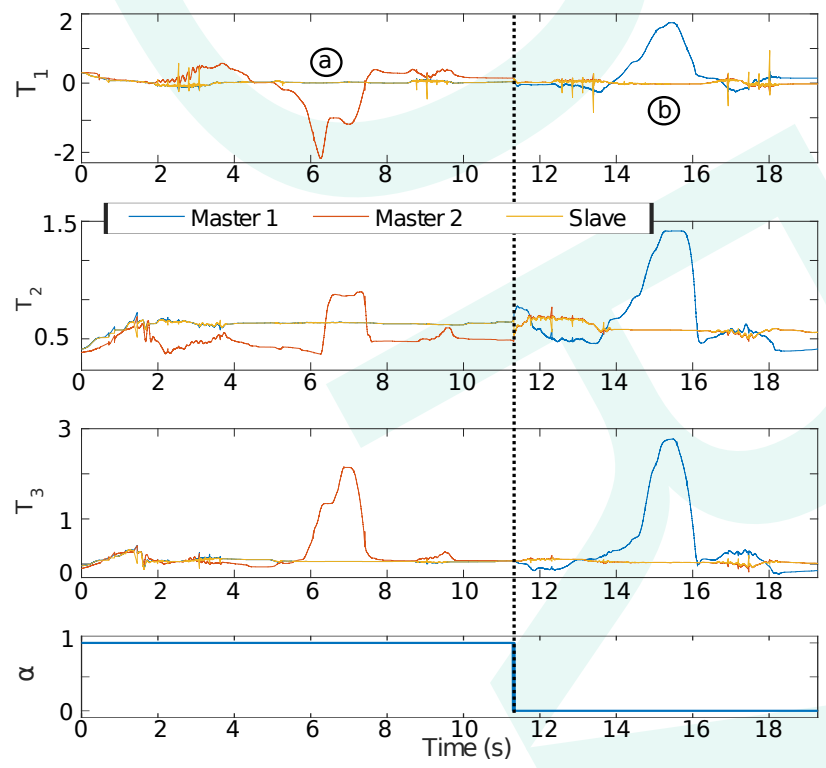

Figure 8: Torques for the three joints and evolution of $\alpha$

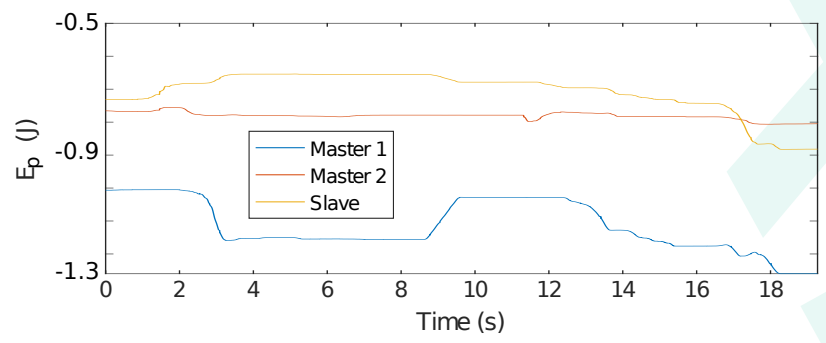

Figure 9: Energy $E_{p}$ generated by both MFS for each joint in free motion

\subsection{Interaction in Contact}

In this second experiment, the dominance factor $\alpha$ is kept to 1 , corresponding to the demonstration mode where the trainer (with master 1) shows an interaction to the trainee (with master 2). The trajectory is visible in Figure 10. The trainer leads his device to apply the slave effector on a surface which has the mechanical characteristics of human bones (model 15PCF from Crea Plast ${ }^{2}$ ), in order to show the behavior of the system in contact with the stiffest organ it may encounter in a medical context. Once in contact, he asks the trainee to come at the same position to be able to feel the same interaction force. This can be very useful

\footnotetext{
${ }^{2}$ See http://www.crea-plast.com/
}

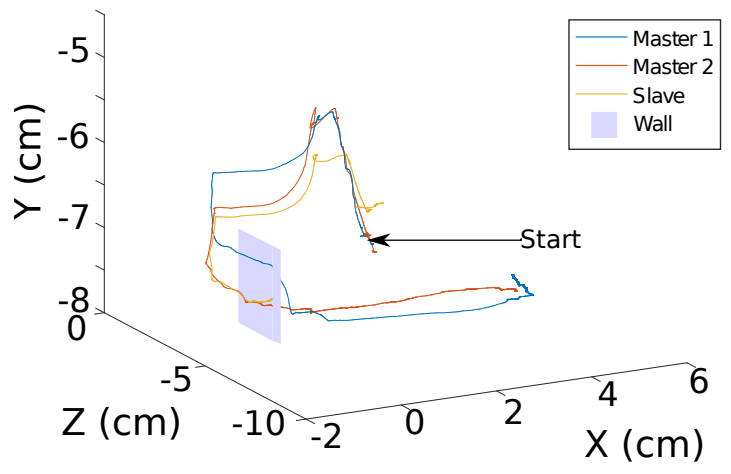

Figure 10: Projection in space of the trajectory performed in contact case

in a training context to learn how to dose forces to apply. As any teleoperation system, bound to a balance between transparency and stability, the positions of the masters are virtually beyond the surface (around $4 \mathrm{~cm}$ ) while the slave effector stopped in contact, which corresponds to a stiffness of $250 \mathrm{~N} / \mathrm{m}$. Below this value, the system can render soft interactions with precision. This stiffness could be raised by using more powerful haptic devices. Increasing the IPC gains would also help but at the price of getting annoying chattering.

Figure 11 shows the position of the effectors along the $x$ axis in cartesian space and the resulting forces in this direction. At $t=3 \mathrm{~s}$, the slave comes into contact with the vertical surface of the piece located at $x=0$ in the plane $y z$. This is visible as the slave position is stuck since. As long as the trainer pushes his own device "inside the piece", the force feedback increases accordingly. In this plot, the slave force overlaps the master 1 feedback force. In steady state, the devices being immobile and as users move along the horizontal $x$ axis, there is no torque disturbance in this direction. In the case of vertical interactions, both torque plots would be shifted together to compensate for the gravity (when touching a surface from bottom). Despite the aforementioned disturbed positioning, the force tracking error is $0.35 \mathrm{~N} \mathrm{RMS}$, corresponding to $3.5 \%$ of the magnitude of the forces). When the trainer decides that this corresponds to the right level of force to apply in a training context, he stops his motion and asks the trainee to join him at the same location, which the latter does at $t \approx 13 \mathrm{~s}$. The follower aligns his own device at the same location as the trainer's (error of $2 \mathrm{~mm}$ RMS in $x$ direction when $t>13 \mathrm{~s}$ ) only by way of a video feedback and real-time plots displayed on his monitor, as visible in Figure 4. He then feels a force feedback which is very close to the interaction force (error of $0.2 \mathrm{~N}$ RMS (2\%) when $\mathrm{t}>13 \mathrm{~s}$ ) as far as we can estimate it. Figure 12 shows that MSF energy levels are not influenced by the contacts.

\section{Conclusion}

For hands-on training purpose, an architecture (Energy Shared Control (ESC)) of a dual user haptic simulator, 5 based on an energetic approach had been previously in- 


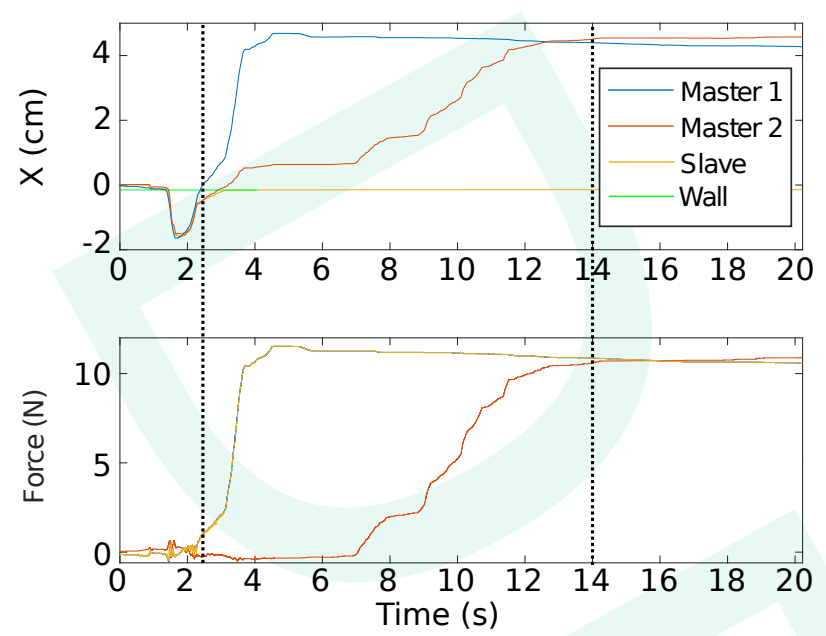

Figure 11: Position and force along cartesian $x$ axis for the trajectory performed in contact case

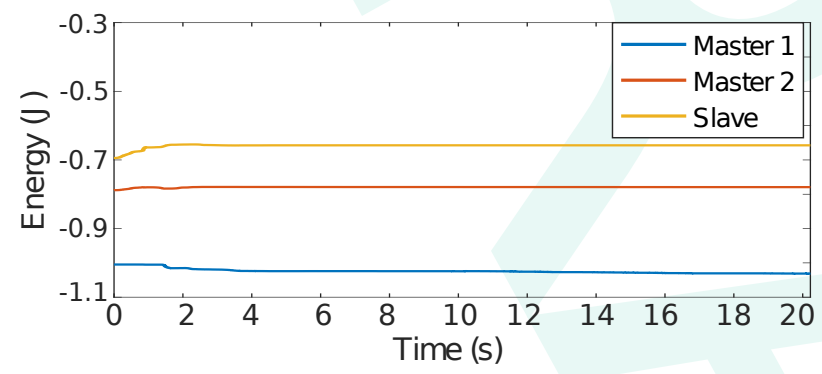

Figure 12: Energy $E_{p}$ generated by both MFS for each joint in contact case

troduced in [1] for only one degree of freedom. In this paper, we extended and experimentally validated it with three dof. This extension only assumes that the three haptic devices are identical. The advantages of this architecture is that the user who observes the person manipulating the tool is guided to follow the same trajectory, and they can feel the same level of forces applied by the tool on its environment simply by moving their own haptic device at the same position. From a didactic point of view, it is a significant property for hands-on training where the dosing of applied efforts is as important as the tool trajectories (in medical domain, for instance). Experiments showed a position tracking precision around one centimeter with a good force tracking. A weight compensation approach should be envisaged to increase the precision of this system as the joint 2 , which supports the whole arm weight, has clearly some difficulties to correctly track the devices. Also tests with more powerful haptic interfaces should be performed to check that we can reproduce interactions with higher stiffness and obtain better precision during interactions. Future works will also consist of pro- viding a solution to use haptic devices with different kinematics and a solution to help following user to align his haptic interface with the one of the leading user.

The authors acknowledge the financial support of the Consejo Nacional de Ciencia y Tecnologia (CONACyT) in Mexico.

\section{References}

[1] F. Liu, A. Lelevé, T. Redarce, D. Eberard, A Dualuser Teleoperation System with Adaptive Authority Adjustment for Haptic Training, in Proceedings of the 4th International Workshop on Medical and Service Robots (MESROB) (Nantes, France, 2015)

[2] C. Sutherland, K. Hashtrudi-Zaad, R. Sellens, P. Abolmaesumi, P. Mousavi, IEEE Transactions on Biomedical Engineering 60, 3009 (2013)

[3] L. Panait, E. Akkary, R.L. Bell, K.E. Roberts, S.J. Dudrick, A.J. Duffy, Journal of Surgical Research 156, 312 (2009)

[4] N. Vaughan, V.N. Dubey, T.W. Wainwright, R.G. Middleton, Medical Engineering \& Physics 38, 59 (2016)

[5] B. Zevin, R. Aggarwal, T.P. Grantcharov, Journal of the American College of Surgeons 218, 294 (2014)

[6] S.S. Nudehi, R. Mukherjee, M. Ghodoussi, IEEE Transactions on Control Systems Technology 13, 588 (2005)

[7] B. Khademian, K. Hashtrudi-Zaad, The International Journal of Robotics Research 30, 1627 (2011)

[8] F. Liu, A. Lelevé, D. Eberard, T. Redarce, An Energy Based Approach for Passive Dual-user Haptic Training Systems, in Proceedings of the 2016 IEEE International Conference on Intelligent Robots and Systems (IROS) (Daejeon, South Corea, 2016)

[9] A. Ghorbanian, S.M. Rezaei, A.R. Khoogar, M. Zareinejad, K. Baghestan, ISA Transactions 52, 268 (2013)

[10] A. Zakerimanesh, F. Hashemzadeh, A.R. Ghiasi, ISA Transactions 68, 33 (2017)

[11] S. Stramigioli, in Lecture Notes in Control and Information Sciences (Springer, 2001), Vol. 266

[12] M. Van Damme, P. Beyl, B. Vanderborght, V. Grosu, R. Van Ham, I. Vanderniepen, A. Matthys, D. Lefeber, Estimating robot end-effector force from noisy actuator torque measurements, in Proceedings of the IEEE International Conference on Robotics and Automation (ICRA) (2011), pp. 1108-1113

[13] C.I. Aldana, E. Nuño, L. Basañez, E. Romero, Journal of the Franklin Institute 351, 1517 (2014)

[14] S. Stramigioli, Creating Artificial Damping By Means of Damping Injection, in Proceedings of the ASME Dynamic Systems and Control Division (1996), Vol. DSC.58, pp. 601-606 